\title{
AUTOMATED InNOVATIVE WHEELCHAIR
}

\author{
Mohammed Asgar, Mirza Badra, Khan Irshad and Shaikh Aftab \\ Department of Electronics and Communication Engineering, Shri Sant Gadgebaba \\ College of Engg. and Tech. Bhusawal, Maharashtra, India
}

\begin{abstract}
New development in sensors, radar and ultrasonic technologies has proved to be a boon for electronics travelling aids (ETAs). These devices are widely used by blind and physically challenged peoples. C5 laser cane, Mowat sensor, belt and binaural sonic aid, NAV guide cane are among popular electronic travelling aids used by blind peoples. For physically challenged person electric wheel chairs controlled by joystick, eye movement and voice recognition are also available but they have their own limitation in terms of operating complexity, noise environment and cost. Our paper proposes an automated innovative wheelchair controlled by neck position of person. It uses simple LEDs, photo sensor, motor and microcontroller to control the movement of wheelchair.
\end{abstract}

\section{KEYWORDS}

Joystick, Sensor, Transmitter, Receiver.

\section{INTRODUCTION}

Electric power wheelchairs have become increasingly important as assistive technology and rehabilitation device and the number of users has grown considerably. Normally wheel chairs are controlled by joystick; however this may not suitable for some disabled people. In modern technology, the HMI based techniques include joysticks controller, finger movement, voice recognition, eye-gaze tracking, electro-myography, etc. These technologies are already included in wheelchairs which are made for the purposes. But proposed wheelchair include a newly invented technology termed as AIW (Automated Innovative Wheelchair).

Handicap person cannot operate joystick wheelchair, whereas in eye tracking wheelchair operator is not free to visualize the adjacent surroundings when system is active, and voice recognition based wheelchair cannot be operated properly in noisy environment. The proposed wheelchair is specially designed for the purpose of eliminating high cost and also for those who are amputees. Automated innovative wheelchair is a wireless system designed for the physically handicapped person specially or the quadriplegic patients. This is a type of moving unit which even can be easily driven by those who do not have arms or legs or both. Neck-movement of the patient will control the motion of the wheelchair. It incorporates LEDs and photo-sensors to accomplish this task.

LEDs and Phototransistors give better response in all environments. This Proposed chair can be operated in almost all kinds of environments as these components are less sensitive to environmental variations. The motion of the neck after exceeding the set value will generate the command signal. Command signal generated at the transmitting end is checked at the receiver side thrice in order to find whether the received signal is command or noise. If any of the received 
three signals is mismatched, then the command is discarded. The additional features include obstacle detection \& pot-hole detection, thereby self-ceasing in both the circumstances, alarm to the user in case of such hazards.

\section{MAterial AND MethodS}

\subsection{A typical joystick controlled wheelchair}

Controllers are most commonly an arm-rest mounted joystick which may have additional controls to allow the user to tailor sensitivity or access multiple control modes. The controller may be swing-away to aid in side-transfers. For users who are unable to use a hand controller various alternatives are available such as sip-and-puff controllers, worked by blowing into a sensor. In some cases the controller may be mounted for use by an aide walking behind the chair rather than by the user. Capabilities include turning one drive wheel forward while the other goes backward, thus turning the wheelchair within its own length [1].

Joystick controlled Power chairs may be designed for indoor, outdoor or indoor/outdoor use. A typical indoor power chair will be narrow and short, to enable better manoeuvring around tight environments [2].

\subsection{Finger Movement Tracking Wheelchair}

In this system, the wheelchair can be controlled by a combination of three fingers. One finger controls the speed of the wheelchair while the other two fingers control the directions of the wheelchair. There is flexibility that we can use only two fingers instead of three to control the direction while letting the system to control the speed automatically. For the purpose of tracking the movement of fingers, the flex-sensors are used. The finger movement tracking system revolves around flex sensor. Flex sensor is basically a resistive strip whose resistance is directly proportional to bending. The flex sensor is worn on the finger and the bending of the finger, in turn, bends the resistive strip thereby increasing the resistance of the strip. This change of resistance is used in generation of command signals [3].

\subsection{Wheelchair based on voice recognition}

In order to benefit the end users who have lost control of their upper extremities due to injury, illness or disability, a speech recognition system is designed to be one of the steering control components of the MWA [4]. The user can communicate with this type of system by giving voice command. This voice command is predefined for each operation supported by the wheelchair. Suppose, if operator is willing to go towards right side then he/she just has to say right and after receiving this command the wheelchair will go in right direction. Similarly, other commands such as start, stop, etc. can be given.

\subsection{Eye tracking based wheelchair}

When the case is worse all other technique becomes useless. In some critical cases, in which the person is unable to move the parts of the body even unable to speak in such cases eye controlled wheelchair can be very effective. In this case, the different commands for the wheelchair are derived from the electrooculography (EOG) potential signals of eye movements. A system for electric wheelchair control using the eyes was proposed in 2007 [5]. A commercially available web camera on a head-mounted display (HMD) which the user wears is used to capture moving pictures of the user's face. A computer mounted on the electric chair processes the captured 
image data, detecting and tracking movements of the user's eyes, estimating the line-of-sight vector, and actuating the electric wheelchair in the desired direction indicated by the user's eyes.

\subsection{EMG based electric power wheelchair}

EMG based EPWs control can be separated into two main groups, Pattern recognition and nonpattern recognition. In pattern recognition-based EMG systems a classifier is used to identify or recognize signal patterns that are extracted from raw data in the form of features [6]. The pattern recognition method depends directly on the features selected and the extraction and classification methods, of which there are variety [7]. This variety causes a problem for researchers in identifying the optimum combination of extraction and classification methods. In recent times, a number of classification methods have been used in an effort to improve the quality of the control of EPWs [8].

\section{Proposed Wheelchair System}

\subsection{Transmitter Section}

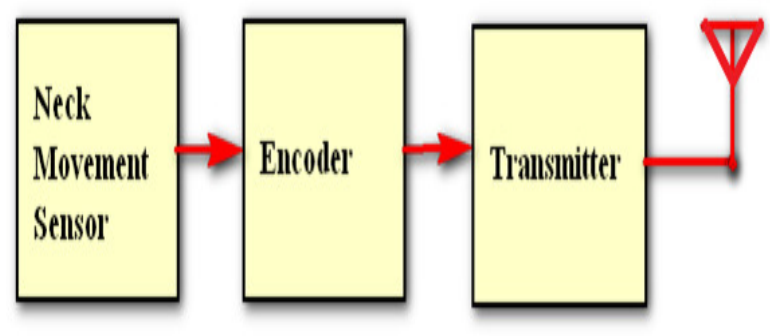

Figure:1 Transmitter section

Transmitter section consists of neck movement sensor, encoder, and transmitter. Depending on these assemblies the control signal is generated for the wheelchair.

\subsubsection{Neck movement sensor}

Most sensors are electrical or electronic, although other types exist. A sensor is a type of transducer. Sensors are either direct indicating (e.g. a mercury thermometer or electrical meter) or are paired with an indicator (perhaps indirectly through an analog to digital converter, a computer and a display) so that the value sensed becomes human readable. In addition to other applications, sensors are heavily used in medicine, industry and robotics. Technical progress allows more and more sensors to be manufactured with MEMS technology. In most cases this offers the potential to reach a much higher sensitivity. There are various way to track movement of neck like ultraviolet, infrared, switches etc. But we have selected infrared sensor due to their better performance. In infrared sensor photodiode detect the incoming signal due to absorption phenomenon.

In our system we will provide three points at which LED's and photodiodes/transistors can align to generate one of the control signal which is predefined. The user will be able to give two commands to this wheelchair i.e. right and left. For controlling the straight direction there is no additional sensor. For moving the wheelchair in the forward direction you just have to align any of the LED and photodiode/phototransistor and look straight. And for stopping it just bow the head for two seconds. After stopping the wheelchair user is free to move his neck but have to take 
care that light should not fall on any of the photo sensors else the wheelchair will turn on. In short there is restriction on neck movement. But for user convenience we have taken care that will permit the user to move the neck around $60^{\circ}-70^{\circ}$ degree in either direction.

\subsubsection{Encoder}

The encoder used is HT12E. When there is any movements in neck then the signal is generated which needs to be transmitted. To transmit the signal efficiently encoder is used. There are number of sensors in the unit and hence the signals from all the sensors must be transmitted and this task is accomplished by the encoder section. Basically it is a parallel to serial converter. It receive number of data at same time and it convert these all signal and give serial output. Parallel to serial conversion rate depend on oscillator frequency or clock frequency. To reduce complexity we have used the device which is having internal oscillator, and we can control the frequency by changing resistance value. Encoder is nothing but series of flip flop which stores data in parallel manner and give output serially. In every encoder there is one terminal enable pin. Whenever terminal enable pin is active then encoder performs encoding operation otherwise it doesn't care about any input.

\subsubsection{Transmitter}

To transmit the encoded signal transmitter is necessary. Selection of transmitter depends on the type of input or whether input is digital signal or analog signal. If the signal is analog then we can use either amplitude modulation(AM) or frequency modulation(FM). And if the encoded signal is digital in nature then we can use amplitude shift keying(ASK), Frequency shift keying(FSK), Quadrature phase shift keying(QPSK), phase shift keying(PSK),etc. In our system encoded data is in digital form hence we have used amplitude shift keying (ASK). The transmitter used in the system fulfils all our requirements such as range data transfer rate etc. There are various ASK module available in Market and it operates in VHF/UHF and ISM bands.

\subsection{Receiver Section}

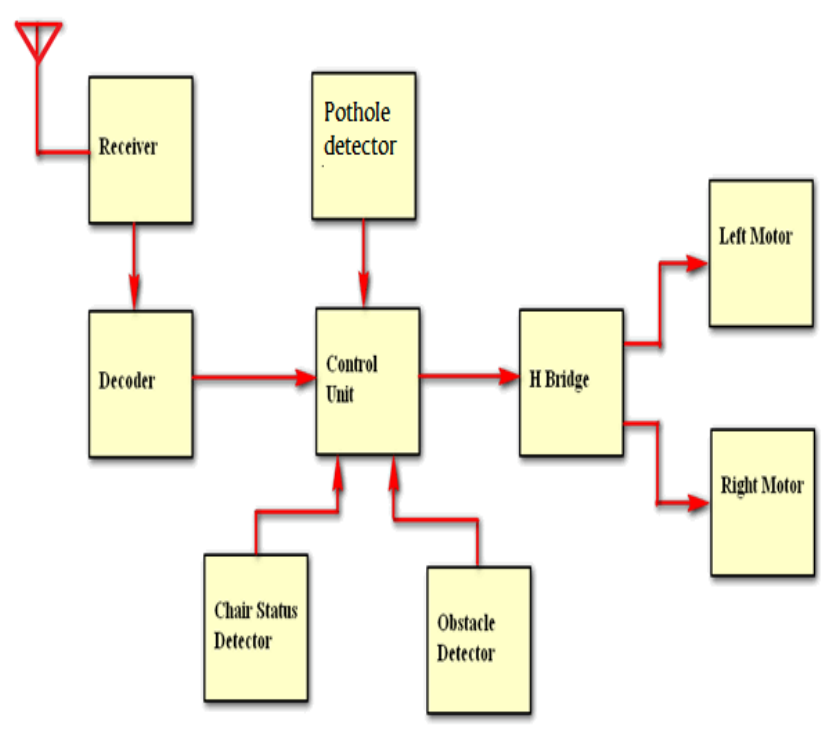

Figure:2 Receiver section 


\subsubsection{Receiver}

It receives the signal transmitted by the transmitter. Receiver performs demodulation operation after receiving signal. Tune frequency of transmitter and receiver must be same. At the receiving antenna numbers of waves are present but due to tuning the desired frequency signal are selected and all other signals are rejected. In market various RF modules are available which gives good performance.

\subsubsection{Decoder}

The decoder used is HT12D. It may happen that more than one transmitter can come in the range of the receiver. Hence with the intention to avoid the confusion at the receiver side addressing technique is used. This encoder and decoder have 8 bit address i.e. 256 combinations of address can be generated. The receiver will receive the data from the transmitter with which its address matches. As the number of data converted or encoded in serial form and transmitted by the transmitter. At receiver it is necessary to convert the data back into the original form which was before encoding. To do so decoder is used. Decoder is nothing but series of flip flop it receives data serially and stores it. When all the flip flops are loaded then the data is made available at the output in parallel manner. To ensure proper decoding encoding rate and decoding rate must be same.

\subsubsection{Pothole detector}

To avoid any accident numbers of provision are taken in wheelchair. This Wheelchair is partially autonomous, it can take many decision by itself. To avoid any damage due to potholes, sensors are installed in wheelchair. There are various way to detect obstacles. We can use ultrasonic Transceiver. In ultrasonic Transceiver signal is transmitted by the transmitter and the time taken by the receiver to receive the reflected signal is proportional to the depth of pothole. Other way to detects potholes is infrared Transceiver. In our system we are using infrared transceiver. The distance from which infrared can reflect is dependent on the bias voltage applied to it. We have used this principal to detect potholes. For this infrared is placed near the wheel. The infrared is biased to a voltage to obtain reflection continuously from the ground. Whenever there is increase in distance the reflection ceases and this interrupts the control units which will halt the system.

\subsubsection{Obstacle detector}

For further safety purpose there is a provision in the wheelchair to detect the obstacles and take the safety decisions. Obstacle detector will detect any obstacle in front of wheelchair. When it finds any obstacle it interrupts the control unit to take desire action. There are two way to detect obstacle either ultrasonic method or infrared method. We are using ultrasonic transceiver module SL HC SR 04. The speed of the ultrasonic wave produced by this module is $29.034 \mathrm{usec} / \mathrm{cm}$. On providing a pulse of 10usec this module transmits 8 pulses at a frequency of $40 \mathrm{KHz}$ this pulse are reflected back and the ranging of the obstacle is done on the basis of time taken by the reflected pulses to reach the receiver. Depending on time as the wave proceeds from the transmitter and gets reflected back to the receiver this module generates a high pulse on its echo pin. The maximum distance from which this wave can reflect is $17 \mathrm{~m}$ at an arc of 7.5 degree on either side. In our system we have decreased this range to $1 \mathrm{~m}$. To achieve this we have programmed our system accordingly. We have set one reference time which is the time required to obtain the reflection from a distance of $1 \mathrm{~m}$. The $10 \mathrm{usec}$ pulse and the reference timer is triggered almost at the same instant. If at the end of this reference time we are still obtaining a high pulse on echo pin that means the obstacle is not in the range of $1 \mathrm{~m}$ and $10 \mathrm{usec}$ pulse is applied again after the echo pin goes low(i.e. after the time interval which is required to obtain the reflection from a distance 
of $17 \mathrm{~m}$ ).And if we are obtaining a low on this pin that means there is an obstacle in the range of $1 \mathrm{~m}$ so the interrupt is generated which will halt the system and allow the user to move either right or left.

\subsubsection{Chair status detector}

For better efficiency and power saving this detector will be used. The function of this detector is to detect whether the person is on the wheelchair or it is vacant. Whenever any person sits on wheelchair then this detector will send signal to the control unit and it will activate the system else, the system will go into power saving mode i.e. sleep mode. Here we have used micro switches as a status detector.

\subsubsection{Control unit}

It's a brain of all system. Control unit can be a microprocessor or microcontroller. It is the intelligence part of the unit which collects the data from each sensor or detector and performs different computation on data and according to the result of different computation it takes decision. It controls the motor speed and direction by $\mathrm{H}$ Bridge methodology depending upon program store in it. Crystal frequency must be adjusted to ensure better performance. We have used microcontroller in our wheelchair because it provides on chip memory and better flexibility.

\subsubsection{H Bridge}

To drive the DC motor in forward and reverse direction $\mathrm{H}$ Bridge is necessary. Normally $\mathrm{H}$ Bridge is made by using relays. Four relays are connected in bridge it looks like an English alphabet $\mathrm{H}$ and hence it is called $\mathrm{H}$ Bridge. It also provides isolation. If we make $\mathrm{H}$ Bridge by using relays for driving a motor it will isolate the high voltage side from the low voltage side and thus it protects damage due to freewheeling action.

\subsubsection{Motor}

Movement in wheelchair is done by motor. Motor is responsible for movement of wheel chair. Motor will be selected depending upon requirement i.e. maximum load, starting torque, voltages ratings, current ratings, etc. In our system we have used two motors.

\section{IMPLEMENTATION}

The mentioned design will be implemented by making the use of components listed below

1] Neck movement sensor: For detecting any movement of neck we have used led's and photodiode in proper arrangement.

2] Encoder and Decoder: For encoding and decoding purpose IC HT12E and HT12D is used.

3] Transceiver module: For wireless transmission and reception purpose SM TX-433 AM/ASK and SM RX-433 AM/ASK module is used respectively.

4] Obstacle detector: To detect any obstacle coming in the path of wheelchair ultrasonic transceiver moduleSL-HC-SR04 is used.

5] Potholes detector: To avoid any shocks due to potholes infrared proximity transceiver module is used.

6] Relays: HUI KE HK19F-DC 5V SHG is the relay which has been used to form H-Bridge for driving the motors.

7] Relay driving IC: IC ULN2803 is used for driving the relays. 
8] Motors: For moving the wheelchair DC motor MY1016Z2 is used.

9] Controlling unit: Microcontroller 89V51RD2 is used for controlling and commanding the wheelchair by programming it accordingly.

\section{Conclusions}

Controlling technique in the proposed system is unique and it is quite simple. Any type of disabled person will able to operate this wheelchair except those who are unable to turn their neck. This proposed system could be a boon for disables. This wheelchair can be made more flexible in future by upgrading the technologies used.

User can give only 4 command signal to the wheel chair, which are start, stop, left and right. This wheelchair will also provide $360^{\circ}$ rotation. This wheelchair is also provided with some intelligence with the help of which, number of decisions can be taken by wheelchair itself. This wheelchair is inspired from Stephen Hawking's wheelchair. In Stephen hawking's wheelchair the electromagnetic rays that are generated by the mind is absorb and depending upon these rays decision is taken.

But this process is extremely costly near about 4 to 5 lakhs investments are needed. And that's why we have designed the automated innovative wheelchair which is comparatively cheap and can be afforded by poor people. Our wheelchair is almost a substitute for Stephen hawking's wheelchair. But, the difference is in the controlling methods i.e. we have used the LED's and photo sensors. This paper could be a great gift for the physically disabled if introduced at industrial level with affordable cost. It could be more than a leg for handicapped with of course no pain since the system is automatic controlling the movement.

\section{REFERENCES}

[1] Thomas R*Ofer And Christian Mandel And Tim Laue, June 23-26, In 2009 IEEE 11th International Conference on:"Rehabilitation Robotics" Kyoto International Conference Center, Japan,

[2] V.I. Pavlovic, R. Sharma, And T.S. Huang, "Visual Interpretation Of Hand Gestures For HumanComputer Interaction: A Review", In IEEE transactions On Pattern Analysis And Machine Intelligence, July 1997,Vol. 19, Pp. 677-695.

[3] Fahad Wallam And Muhammad Asif, August 2011, In International Journal Of Computer And Electrical Engineering, on: "Dynamic Finger Movement Tracking And Voice Commands Based Smart Wheelchair", Vol. 3, No. 4.

[4] Mazo, M., Rodrfguez, F.J., Lfizaro, J.L., Urefia, J., Garcia, J.C., Santiso, E., Revenga, R, and Garcia, J.J. 1994b. Intelligent Electronic Control for a Wheelchair Guided by Voice Commands and External Sensors. AIRTC'94. pp. 385-390. Valencia (Spain).

[5] Arai K, Purwanto D, December 2007, In 7th International Conference On Optimization: on "Techniques And Applications of Electric Wheelchair Control with the Human Eye". Kobe, Japan

[6] I. Moon, M. Lee, J. Chu, And M. Mum, "Wearable EMG-Based HCI For Electric-Powered Wheelchair Users With Motor Disabilities," In IEEE Int. Conf. Robotics And Automation,Barcelona,Spain, Pp.2649-54,Apr.2005.

[7] L. Wei, H. Hu, And K. Yuan, "Use Of Forehead Bio-Signals For Controlling An Intelligent Wheelchair,"In IEEE Int. Conf. Robotics And Biomimetics, Bangkok, Thailand, Feb.2008 Pp.108-13,

[8] M. A. Oskei And H. Hu," My electric Control System-A Survey", In Biomed. Signal Process. Control, Oct 2007. Vol.2,No.4. Pp.275-94. 
International Journal of Information Technology Convergence and Services (IJITCS) Vol.3, No.6, December 2013

\section{Authors}

Mohammed Asgar completed Diploma in Industrial Electronics engg and currently pursuing degree in Electronics Communication Engineering From S.S.G.B.C.O.E.T.Bhusawal.

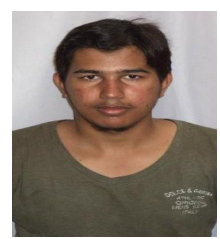

Mirza Badra completed Diploma in Industrial Electronics engg and currently pursuing degree inElectronics \& Communication Engineering From S.S.G.B.C.O.E.T.Bhusawal

Khan Irshad completed Diploma in Electronics engg and currentlypursuing degree In Electronics \& Communication Engineering From S.S.G.B.C.O.E.T.Bhusawal.
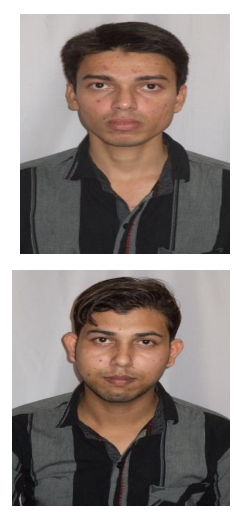

Shaikh Aftab completed Diploma in Electronic \& Telecomm engg and currently pursuing degree inElectronics \&Communication Engineering From S.S.G.B.C.O.E.T.Bhusawal.

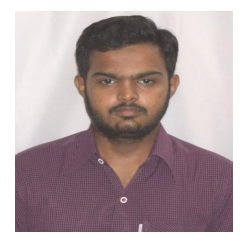

